\title{
Robust optimization for U-shaped assembly line worker assignment and balancing problem with uncertain task times
}

\author{
Ömer Faruk Yılmaz ${ }^{1, *}$ \\ ${ }^{1}$ Department of Industrial Engineering \\ Karadeniz Technical University, Trabzon, Turkey \\ E-mail:〈omerfarukyilmaz@ktu.edu.tr〉
}

\begin{abstract}
Awareness of the importance of U-shaped assembly line balancing problems is all on the rise. In the U-shaped assembly line, balancing is affected by the uncertainty associated with the assembly task times. Therefore, it is crucial to develop an approach to respond to the uncertainty caused by the task times. When the great majority of existing literature related to uncertainty in the assembly line is considered, it is observed that the U-shaped assembly line balancing problem under uncertainty is scarcely investigated. That being the case, we aim to fill this research gap by proposing a robust counterpart formulation for the addressed problem. In this study, a robust optimization model is developed for the U-shaped assembly line worker assignment and balancing problem (UALWABP) to cope with the task time uncertainty characterized by a combined interval and polyhedral uncertainty set. A real case study is conducted through data from a company producing water meters.
\end{abstract}

Keywords: robust optimization, uncertainty, U-shaped assembly line balancing, worker assignment

Received: April 15, 2020; accepted: November 16, 2020; available online: December 18, 2020

DOI: $10.17535 /$ crorr.2020.0018

\section{Introduction}

The U-shaped assembly line balancing is one of the well-known problems in the existing academic literature. Because the U-type layout is proved to be more effective than the straight line concerning efficiency and flexibility, many studies have been conducted on this area [11]. In the context of U-shaped assembly line balancing, there are models developed for situations where the parameters or the probability distribution of the parameters are fully known. However, a great majority of these studies have not considered the uncertainty and vagueness associated with assembly task times in real production systems but focused on the deterministic environment. In this study, we focused on the U-shaped assembly line balancing problem since this type of layouts is commonly used in many industries. To this end, a robust optimization model is developed to balance U-shaped assembly lines under the uncertainty of task times. The proposed optimization model leads to obtain robust solutions for balancing the U-shaped line with uncertain task times.

The uncertainty in the parameters is generally handled with two approaches (i) stochastic optimization and (ii) robust optimization. Stochastic optimization describes the uncertainty with probability [24]; however, the parameter uncertainty is set-based and the optimization model is deterministic in the robust optimization [5]. Therefore, robust optimization allows addressing the parameter uncertainty more comprehensively compare to stochastic optimization, in which the scenario-based approach is applied [17]. Besides, a robust approach generally leads

${ }^{*}$ Corresponding author. 
to a computationally tractable solution, which is one of the important issues for optimization [3]. In robust optimization, a conservative procedure is conducted by taking the possible realization of uncertain parameters into account. By doing so, a robust solution can be achieved from suboptimal solutions to guarantee the optimality of the solutions without encountering overconservativeness in case of parameter change. It should be considered that the implemented robust approach and uncertainty set have a considerable impact on the conservativeness of the solutions. The noteworthy points for robust optimization are (i) uncertainty set, (ii) tractability, and (iii) conservativeness and probability of constraint violation, (iv) complexity of the model, and (v) quality of the solution [5, 22]. While implementing robust optimization, there is no need for historical data to estimate the distributions. Besides, the robust optimization focuses on the worst-case scenario and thereby the strict constraints are included in the optimization model. On the other hand, stochastic optimization considers the expected values of all possible scenarios by considering the data at hand. Therefore, robust optimization is preferred as seeking the solution for the worst-case scenario in case of the absence of probability information.

Two important issues should be considered for robust formulation (i) tractability and (ii) conservativeness with probability guarantees. Tractability is related to the solvability of the robust model and obtaining tractable solutions depends on the nominal problem and the uncertainty set. The selection of a robust approach is important to avoid conservative solutions and therefore several formulations following uncertainty sets are proposed in the academic literature. See $[3,22]$ for example. In addition, the probability of constraint violations can be computed in advance as a function of the structure of the uncertainty set [5].

In this study, a robust optimization model is developed to solve the single model U-shaped assembly line worker assignment and balancing problem (UALWABP) considering the task time uncertainty. The robust model is developed based on the model introduced by $[15,23]$ for the deterministic single model UALWABP problem. The major contribution of this study, to the best of the authors' knowledge, a robust optimization approach is employed for the UALWABP problem for the first time in the existing literature. Moreover, a real case study from a manufacturing company producing water meters is presented to show the applicability of the proposed model. The price of robustness associated with the solutions is explained based on the computational results.

The rest of the paper is organized as follows. A comprehensive literature review on the robust optimization for the assembly line balancing problem is presented in Section 2. The problem statement and optimization model are provided in Section 3. The problem data with the case study is presented and the computational studies are conducted in Section 4. Concluding remarks and future research directions are given in Section 5 .

\section{Literature review}

In this section, we reviewed the studies conducted for the application of a robust optimization approach on the assembly line balancing problem, in particular U-shaped assembly line balancing.

While many studies conducted to cope with the uncertainties in task time for assembly line balancing used stochastic programming and fuzzy logic $[1,2,7,8,16]$, relatively few studies investigated practical applications of robust optimization for dealing with uncertainties in assembly line balancing, in particular U-shaped assembly line, see for example $[6,9,11,13,18,20]$.

Robust optimization is proposed in the early 1970s by [21]. Since then, it has been effectively used to handle uncertain data in optimization problems [4]. There are several approaches for applying robust optimization, see [3, 22] for example.

$\mathrm{Li}$ et al. [12] introduced robust optimization approaches for mixed-model assembly line balancing problems subject to task time uncertainty. They developed a genetic algorithm-robust 
methodology for the problem. Gurevsky et al. [9] addressed the robust balancing for straight assembly lines. A breadth-first search procedure was employed to obtain optimal solutions. Hazır and Dolgui [10] presented a robust approach with an optimization model by considering interval uncertainty of task times in assembly lines. A decomposition-based algorithm was developed for large-sized problem instances. Moreira et al. [13] focused on a robust assembly line balancing problem with the objective of the minimal number of stations where heterogeneous workers are assigned. Hazır and Dolgui [11] proposed a robust approach for the U-shaped assembly line balancing problem for the first time by considering worst-case situations. Pereira [18] studied an interval data version of the min-max regret (robust) based assembly line worker assignment and balancing problem (ALWABP). Pereira and Álvarez-Miranda [19] developed a robust formulation for the task-time uncertainty of the assembly line balancing problem. The cost of protecting a solution in case of uncertainty was investigated through the experiments. Chica et al. [6] used robust multi-objective optimization and simulation method to obtain robust solutions for assembly line balancing problem. A real case study was presented through six different configurations to show the effectiveness robust approach. Samouei and Ashayeri [20] developed two different robust optimization models in which the objective is to minimize both cost and cycle time. Robust solutions were obtained for different confidence levels.

The vast majority of the existing literature related to the robust optimization with the assembly line balancing problem is reviewed in detail. The literature on the role of a robust optimization approach in the assembly line balancing is rather sparse. Therefore, an extensive survey of the literature suggests that there is a need to develop a robust optimization model to consider such balancing problems. This study contributes to the U-shaped assembly line balancing literature by providing a comprehensive robust optimization model for UALWABP. On one hand, from the managerial point of view, the proposed model is novel by considering the tactical level decisions for managers. On the other hand, from the application perspective, tractable solutions can be obtained easily via the proposed model.

In this regard, this study is the first attempt to investigate the UALWABP within the context of robust optimization. To this end, a robust optimization model is proposed by carefully investigating the addressed problem subject to task time uncertainty.

\section{Optimization model}

The developed robust optimization model intends to solve the single model UALWABP problem under uncertainty. The considered objective is the minimization of the total number of workstations. The detailed information related to UALWABP can be found in [14, 15, 23].

\section{Assumptions}

- The robust optimization is considered to cope with the uncertainty of task times.

- The assembly line is constructed for one product type.

- The cycle time is known in advance.

- The walking time for workers is neglected.

The optimization model is adapted from $[15,23]$ by extending the original model to include task time uncertainty. The robust linear equivalent of their model is modified according to the uncertainty set suggested by [4]. Only the positive variables are considered by [22]. The robust counterpart of the U-shaped assembly line worker assignment and balancing model is then formulated as follows.

\section{Indices}

$i, r, s$ : Indices of tasks $(i: 1, \ldots, I)$

$j$ : Index of workstations $(j: 1, \ldots, J)$

$w$ : Index of workers $(w: 1, \ldots, W)$

Parameters

$n$ : total number of tasks 
$t_{i}$ : operation time for task $i$

$m_{\max }$ : maximum number of stations allowed to be utilized $\left(m_{\max } \leq n\right)$

$C$ : cycle time $W_{j}$ : set of tasks that can be assigned to station $j$

$\left\|W_{j}\right\|$ : number of tasks in set $W_{j}$

$L(r, s)$ : a set for tasks that preceding task $s$

$\Gamma$ : represent the number tasks with uncertain operation time

$\hat{\mathrm{t}}$ : deviation of parameter $t_{i}$

\section{Variables}

$A_{j w}$ : if worker $w$ is assigned to station $j, 1$; otherwise, 0

$x_{i j}$ : if task $i$ is assigned to station $j$ with original network, 1 ; otherwise, 0

$y_{i j}$ : if task $i$ is assigned to station $j$ with phantom network, 1 ; otherwise, 0

$k_{i j}$ : if task $i$ is allocated to station $j, 1$; otherwise, 0

$s_{i j w}$ : if worker $w$ is allocated to task $i$ in station $j, 1$; otherwise 0

$y_{r j w}$ : auxiliary variable defined for variable $s_{i j w}$

$p$ : auxiliary variable used for parameter $\Gamma$

$q_{i}$ : auxiliary variable used for deviation of parameter $t_{i}$

$z_{j}$ : if station $j$ is utilized, 1 ; otherwise 0

\section{Objective function}

$$
\operatorname{Min} \sum_{j=1}^{J} z_{j}
$$

\section{Constraints}

$$
\begin{gathered}
\sum_{j=1}^{J} k_{i j}=1 \quad \forall i \\
x_{i j}+y_{i j}=k_{i j} \quad \forall i, j \\
\sum_{i=1}^{I} t_{i} \times s_{i j w}+p \times \Gamma+\sum_{i=1}^{I} q_{i} \leq C \times z_{j} \quad \forall j, w \\
p+q_{i} \geq \hat{t}_{i} \times y r_{i j w} \quad \forall i, j, w \\
y r_{i j w} \geq s_{i j w} \quad \forall i, j, w \\
\sum_{j=1}^{J}\left(m_{\max }-j+1\right) \times\left(x_{r j} \times x_{s j}\right) \geq 0 \quad \forall r, s \in L \\
\sum_{j=1}^{J}\left(m_{\max }-j+1\right) \times\left(y_{r j} \times y_{s j}\right) \geq 0 \quad \forall r, s \in L \\
\sum_{i \in W_{j}} k_{i j}-\left\|W_{j}\right\| \times z_{j} \leq 0 \quad \forall j
\end{gathered}
$$




$$
\begin{gathered}
\sum_{w=1}^{W} A_{j w} \leq 1 \quad \forall j \\
z_{j}-\sum_{w=1}^{W} A_{j w}=0 \quad \forall j \\
A_{j w}+k_{i j} \geq 2 \times s_{i j w} \quad \forall i j w \\
A_{j w}+k_{i j} \leq 1+s_{i j w} \quad \forall i j w \\
x_{i j}, y_{i j}, z_{j}, A_{j w}, s_{i j w}, y r_{i j w}, k_{i j} \in 0,1
\end{gathered}
$$

The objective function (1) minimizes the total number of stations in which at least one task is assigned. In this regard, the addressed problem is considered to be UALWABP-1, given the cycle time, minimize the number of stations. Equations (2)-(3) state that each task must be assigned to the front or back of the U-shaped line. In other words, a task must be assigned to the forward or backward of a station. Equation (4) is related to the cycle time of each station and includes the robustness of the model. While $p$ and $q$ are employed as auxiliary variables, $\Gamma$ is used as a robust parameter determining the number of tasks with uncertain task times. Equations (5)-(6) are also added to the model to develop the robust counterpart of the nominal model proposed by $[15,23]$. In these equations, the interval and polyhedral uncertainty set proposed by [4] is considered to obtain tractable and non-conservative solutions. This combined uncertainty set is described as follows:

$$
\varphi_{\text {Int }+P o l}(\Phi, \Gamma)=\left\{\hat{a}_{i j}\|\eta\|_{\infty} \leq \Phi,\|\eta\|_{1} \leq \Gamma\right\}
$$

The parameter $\Gamma$ takes in $[0,|\mathrm{I}|]$ and this interval changes for each constraint. The robust counterpart of the investigated problem is constructed under the interval and polyhedral uncertainty set properties, in which the parameter $\Gamma$ determines the number of tasks subject to uncertainty. When the parameter $\Gamma$ is equal to 0 , the model corresponds to the nominal model, which means that there is not an uncertain task time. The considered uncertainty set for the distribution of task time $(\eta)$ taking values within the bound $\Phi=1$. It is noteworthy to state that $\|\eta\|_{\infty}=1$ corresponds to the over conservative formulation. Under this uncertainty set dynamics, the implementation of the addressed uncertainty set to the optimization model is explained in [22].In equation (16), while $p$ is defined as an auxiliary variable used for parameter $\Gamma, q_{i}$ is considered as an auxiliary variable used for deviation of parameter $t_{i}$. For the investigated problem, the operation times of tasks are uncertain, and therefore the robust optimization modeling approach is used to cope with this situation. The task times $\hat{t}$ are considered to be uncertain and independently distributed and follow a symmetric distribution in $[\mathrm{t}-\hat{\mathrm{t}}, \mathrm{t}+\hat{\mathrm{t}}]$ where $t$ denotes the nominal values of the task times. In a U-shaped assembly line, parameter $\Gamma$ regulates how many tasks' operation times deviate. By doing so, we avoid dealing with over-pessimistic situations. In equation (16), $|\mathrm{I}|$ represents the number of tasks in the U-shaped assembly line. The probability of violation of a constraint is determined by $\exp (-\Gamma / 2 \times|\mathrm{I}|)$. Equations (7)-(8) developed by [23] to represent the precedence constraints for both original and phantom networks. While equation (7) is used for predecessor tasks, equation (8) is employed for the successor tasks of the corresponding task. Equation (9) ensures that when a task is assigned to a station, this station must be constructed. Equations (10)-(12) state that a worker can be assigned to at most one station and similarly a station can be assigned to at most one worker. Equation (10) states that a worker cannot be assigned more than one station. Equation (11) indicates that a station cannot be operated by more than one worker. Equation (12) ensures that only one worker is 
assigned to a station if the corresponding station is utilized. Equations (13)-(14) are used to protect the linear structure of the optimization model by obtaining the piecewise linear function. Equation (15) defines the sign restrictions and binary variables. The optimization model is developed for solving the addressed problem. In this way, (i) worker to station assignment, (ii) task to station assignment decisions are aimed to be made concurrently.

$$
s 1_{j}=\sum_{i=1}^{I} k_{i j} \times t_{i}=0 \quad \forall j
$$

Equation (17) is employed to compute the total operation time of tasks in the $j t h$ station. By doing so, the difference between station operation times can be computed to analyze the impact of the parameter $\Gamma$. As indicated in the introduction, the tractability of a robust model and obtaining less conservative solutions are two important issues for robust optimization. Bertsimas and Sim [4] suggested a new robust approach to control the degree of conservatism with a combined interval and polyhedral uncertainty set. In their approach, the tractability of the model is ensured by constructing a linear model in which only some of the parameters are allowed to deviate. For this reason, we also apply the same approach in this study. In the next section, computational studies are conducted to investigate these issues.

\section{Case study and computational results}

In this section, the proposed optimization model is employed in a real case study by providing data from a company producing water meters. The precedence diagram representing relations between assembly tasks along with the nominal values of task times is shown in Figure 1. There are 16 different tasks in total and the precedence relations among tasks along with nominal operation times are shown in Figure 2. The robust counterpart of the nominal model is constructed with a combined interval and polyhedral uncertainty set as it is proposed by [4].

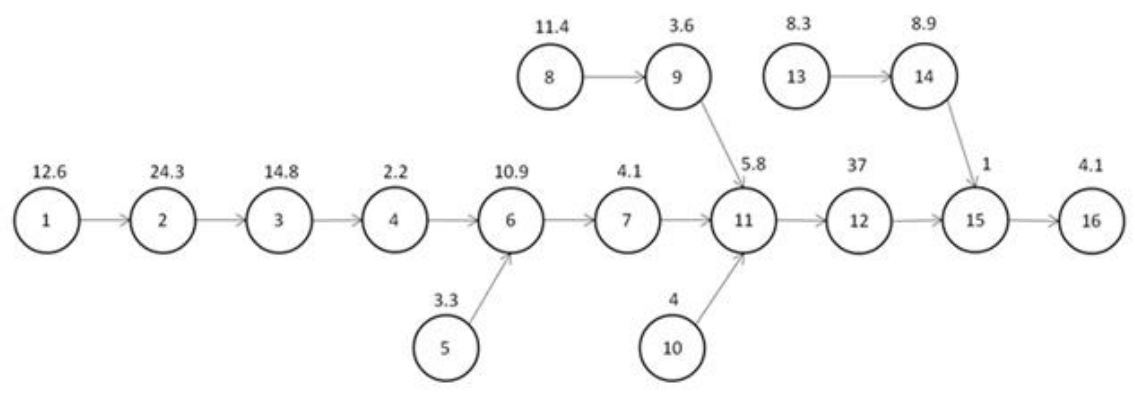

Figure 1: Precedence relationship diagram and nominal values of task times

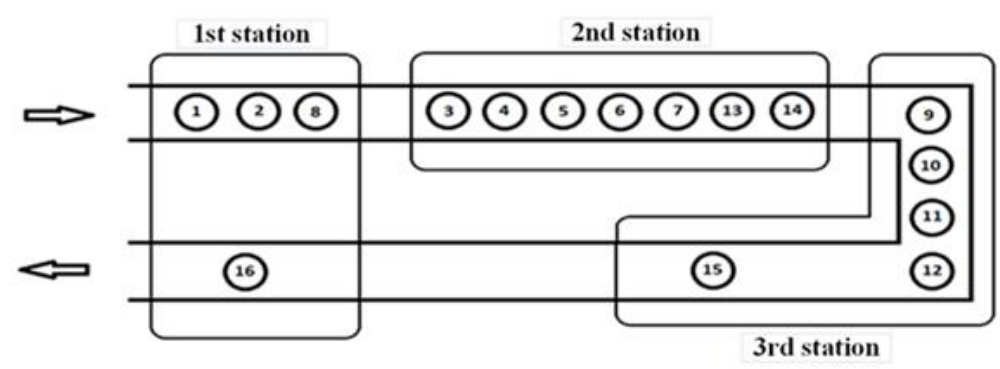

Figure 2: Optimal solution for $U$-shaped assembly line for $\Gamma=0$ 
The task time uncertainty is equal to $5 \%$ of the nominal task times. We assume that only the task operation times are subject to uncertainty. The parameter $\Gamma$ controls the degree of conservatism.

Because it is not likely that all task times change their values simultaneously. Thus, it is plausible to assume that only a subset of task times can vary and affect the objective function. A solution is considered to be robust when $\Gamma$ number of parameters change and it remains feasible, in other words, the solution is robust against the worst-case scenario. For the cycle time constraint (4), we define $\Gamma$ that is always less than the number of uncertain task times. When the parameter is equal to $0(\Gamma=0)$, the model corresponds to the nominal model, which means that there is not an uncertain parameter. In this manner, Figure 2 represents the optimal solution after the proposed model is solved to balance the assembly line for the deterministic case. There are three different stations in the system and each of which is ruled by a worker.

To analyze the problem with different problem specifications, two different cycle time values $(\mathrm{C}=56.1$ and $\mathrm{C}=60)$, three different values of the maximum number of stations allowed in the system $(M \max =5, M \max =8$, and $M \max =11)$, and two different values of the uncertainty level $(2 \%$ and $5 \%)$ are considered. The problem is solved for each parameter combination and the problem data is the same for all runs. A personal computer with hardware of Intel $\mathbb{R}$ CoreTM i7-3630QM CPU and 16 GB memory is used to conduct experiments. The results are obtained for four different $\Gamma$ parameter levels to obtain optimal solutions in a reasonable computation time. The computational results are given in Table 1, in which U:Uncertainty level; MWD: Maximum workload difference; NS: Number of utilized stations; PoV: Probability of the constraint violation; C: Cycle time; Mmax: Maximum number of allowed station.

According to the results, it is observed that the total number of stations utilized in the system (objective function) is not changed with any parameter combination. This is the reason behind why we consider the change in the maximum workload to observe the impact of conservatism degree on the system performance. The most important conclusion that can be deduced from Table 1 is that as the degree of conservatism increases, the workload imbalance decreases. Table 1 shows the optimal values for different parameter settings along with approximate probability bounds of constraint violation. In this table, $\mathrm{C}$ is related to customer orders. While $\mathrm{C}=56.1$ reflects the real data from production, $\mathrm{C}=60$ is determined to make a detailed analysis in case of less demand.

For each scenario of conservatism degree, Figures 3-4 show the change in the maximum workload difference (maximum station workload-minimum station workload) along with the conservatism degree for uncertainty levels $2 \%$ and $5 \%$ respectively. As stated earlier, it can be concluded that when the conservatism increases, the workload difference among stations decreases. In other words, as the number of operation times allowed to change increases, the system instability decreases. This indicates that the robust approach performs better when the system has a high degree of uncertainty.

The price of robustness is the reduction from nominal objective value to robust objective value. When we examine the price of robustness with respect to the maximum workload difference, it is observed that the robust solutions do not lead to worse results compared to the nominal optimal value, which can be obtained for $\Gamma=0$. Thus, the price of robustness is interpreted contrary to the known in the existing literature. From Figure 3 , it is observed that the price of robustness reaches its highest value when $\mathrm{Mmax}=8$ and $\mathrm{C}=56.1$ for $5 \%$ uncertainty of task times, that is, by $97.5 \%$. From Figure 4, it is observed that the price of robustness reaches its highest value when $M \max =5$ and $\mathrm{C}=60$ for $5 \%$ uncertainty of task times, that is, by $89.4 \%$. These results indicate that the price of robustness can change with respect to the different parameter combinations. The cost of protecting the number of stations utilized in the system is equal to 0 , i.e. there is not any cost to protect the assembly line against the uncertainty associated with task times. Furthermore, this cost is negative when the response variable is accepted to be the maximum workload difference. 

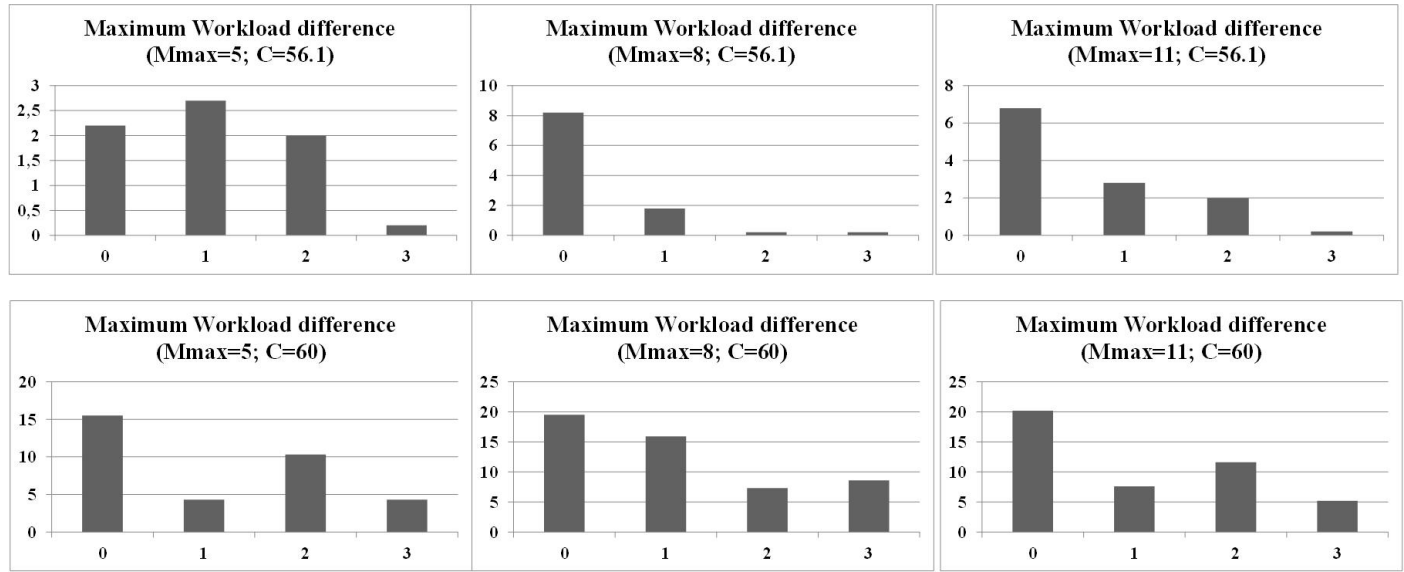

Figure 3: Maximum workload differences for $5 \%$ uncertainty of task times ( $\Gamma$ between 0 and 4 )

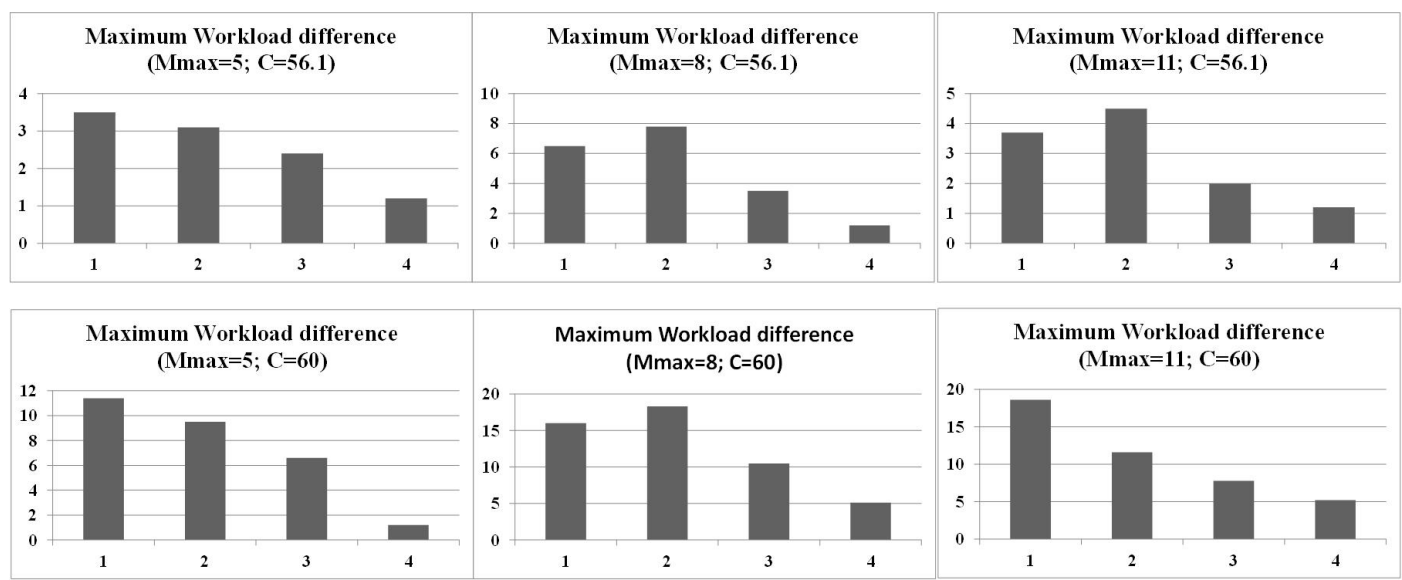

Figure 4: Maximum workload differences for 2\% uncertainty of task times ( $\Gamma$ between 0 and 4)

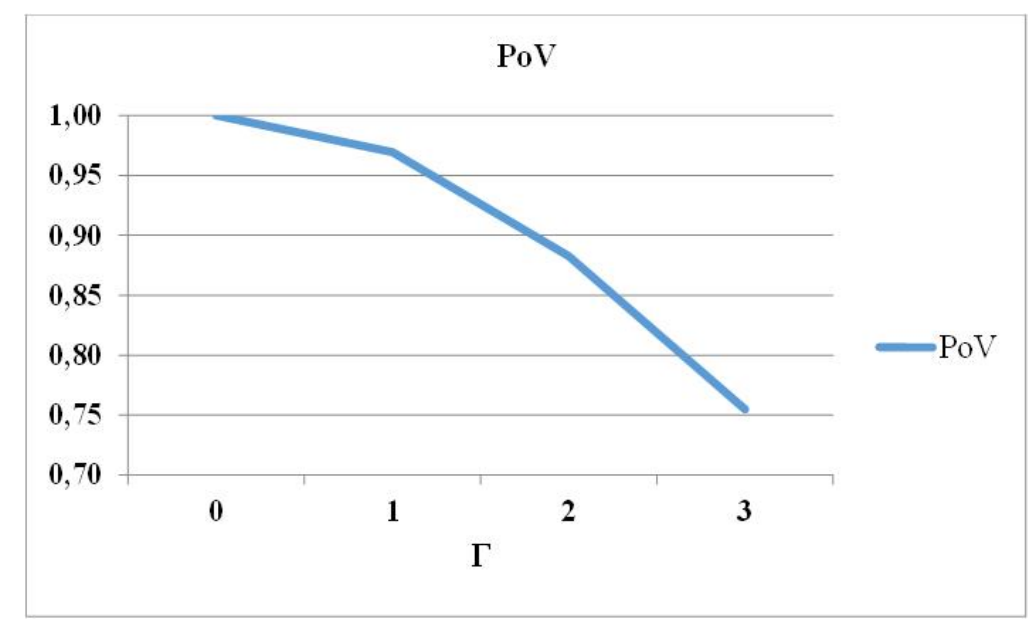

Figure 5: Probability of constraint violation with respect to degree of conservatism 
From Figure 5, it is observed that the probability of constraint violation decreases as the degree of conservatism increases. These values represent the probability bounds for violations and the minimum bound is obtained for $\Gamma=3$. This result indicates that the maximum workload difference does not have to protect the system against constraint violation.

\begin{tabular}{|lllcccclcccccc|}
\hline U-0.05 & & $\Gamma$ & MWD & NS & CPU & PoV & & & $\Gamma$ & MWD & NS & CPU & PoV \\
\hline \hline Mmax & 5 & 0 & 2.2 & 3 & 0.2 & 1.00 & Mmax & 5 & 0 & 15.5 & 3 & 0.7 & 1.00 \\
C & 56.1 & 1 & 2.7 & 3 & 2.8 & 0.97 & C & 60 & 1 & 4.3 & 3 & 7.7 & 0.97 \\
& & 2 & 2 & 3 & 6.7 & 0.88 & & & 2 & 10.3 & 3 & 20.9 & 0.88 \\
& & 3 & 0.2 & 3 & 92.4 & 0.75 & & & 3 & 4.3 & 3 & 70.8 & 0.75 \\
Mmax & 8 & 0 & 8.2 & 3 & 0.9 & 1.00 & Mmax & 8 & 0 & 19.5 & 3 & 0.7 & 1.00 \\
C & 56.1 & 1 & 1.8 & 3 & 1.2 & 0.97 & C & 60 & 1 & 15.9 & 3 & 37.2 & 0.97 \\
& & 2 & 0.2 & 3 & 1.4 & 0.88 & & & 2 & 7.3 & 3 & 50.7 & 0.88 \\
& & 3 & 0.2 & 3 & 117.2 & 0.75 & & & 3 & 8.6 & 3 & 215.8 & 0.75 \\
Mmax & 11 & 0 & 6.8 & 3 & 0.6 & 1.00 & Mmax & 11 & 0 & 20.2 & 3 & 0.8 & 1.00 \\
C & 56.1 & 1 & 2.8 & 3 & 26.1 & 0.97 & $\mathrm{C}$ & 60 & 1 & 7.6 & 3 & 40.6 & 0.97 \\
& & 2 & 2 & 3 & 51.6 & 0.88 & & & 2 & 11.6 & 3 & 56.2 & 0.88 \\
& & 3 & 0.2 & 3 & 169.3 & 0.75 & & & 3 & 5.2 & 3 & 301 & 0.75 \\
\hline U-0.02 & & $\Gamma$ & MWD & NS & CPU & PoV & & & $\Gamma$ & MWD & NS & CPU & PoV \\
\hline Mmax & 5 & 0 & 3.5 & 3 & 1.2 & 1.00 & Mmax & 5 & 0 & 11.4 & 3 & 0.7 & 1.00 \\
C & 56.1 & 1 & 3.1 & 3 & 8.8 & 0.97 & C & 60 & 1 & 9.5 & 3 & 10.7 & 0.97 \\
& & 2 & 2.4 & 3 & 55.7 & 0.88 & & & 2 & 6.6 & 3 & 59.9 & 0.88 \\
& & 3 & 1.2 & 3 & 192.4 & 0.75 & & & 3 & 1.2 & 3 & 160.8 & 0.75 \\
Mmax & 8 & 0 & 6.5 & 3 & 0.9 & 1.00 & Mmax & 8 & 0 & 16 & 3 & 0.7 & 1.00 \\
C & 56.1 & 1 & 7.8 & 3 & 1.2 & 0.97 & C & 60 & 1 & 18.3 & 3 & 37.2 & 0.97 \\
& & 2 & 3.5 & 3 & 1.4 & 0.88 & & & 2 & 10.5 & 3 & 54.7 & 0.88 \\
& & 3 & 1.2 & 3 & 117.2 & 0.75 & & & 3 & 5.1 & 3 & 158.8 & 0.75 \\
Mmax & 11 & 0 & 3.7 & 3 & 0.7 & 1.00 & Mmax & 11 & 0 & 18.6 & 3 & 0.8 & 1.00 \\
C & 56.1 & 1 & 4.5 & 3 & 26.6 & 0.97 & C & 60 & 1 & 11.6 & 3 & 40.6 & 0.97 \\
& & 2 & 2 & 3 & 51.4 & 0.88 & & & 2 & 7.8 & 3 & 56.2 & 0.88 \\
& & 3 & 1.2 & 3 & 169.3 & 0.75 & & & 3 & 5.2 & 3 & 201.5 & 0.75 \\
\hline
\end{tabular}

Table 1: Computational results for different parameter settings

\section{Concluding remarks}

This paper explores the U-shaped assembly line worker assignment and balancing problem (UALWABP) with uncertain task times. To investigate the uncertainty and its impact on performance, a robust optimization approach is employed. That being the case, a robust counterpart formulation is developed for the single UALWABP problem under task time uncertainty. While developing the robust counterpart of the original model, the approach introduced by [4] is considered. Conservativeness and tractability issues are investigated in detail by several parameter combinations. Tractable solutions are found via the proposed model without knowing full knowledge about the distribution of task times. The solution conservativeness is controlled by adjustable parameters $(\Gamma)$ for the combined interval and ellipsoidal uncertainty set. The proposed approach is applied to a real-life case study from a manufacturing company producing water meters. Several managerial insights are obtained based on computational results. (i) The workload imbalance decreases with an increasing degree of conservatism. (ii) The robust approach performs better when the system has a high degree of uncertainty. (iii) There is no 
cost to protect the number of stations utilized in the system against uncertainty associated with task times. (iv) Protection cost is negative when the response variable is accepted to be the maximum workload difference. (v) Overall, we do not have to penalize the objective function or increase workload difference among stations against uncertainty and constraint violation. In the future, this study can be extended in different directions. (i) The scope of the study can be re-analyzed by considering other uncertainty sets such as box, interval, and ellipsoidal. (ii) Various design configurations may be considered for the assembly line and the addressed problem can be investigated in this context. (iii) Last but not least, the robust formulation can be extended for mixed-model U-shaped assembly lines.

\section{References}

[1] Ağpak, K., and Gökçen, H. (2007). A chance-constrained approach to stochastic line balancing problem. European Journal of Operational Research, 180(3), 1098-1115. doi: 10.1016/j.ejor.2006.04.042

[2] Babazadeh, H., Alavidoost, M. H., Zarandi, M. F., and Sayyari, S. T. (2018). An enhanced NSGAII algorithm for fuzzy bi-objective assembly line balancing problems. Computers $\mathcal{E}$ industrial engineering, 123, 189-208. doi: 10.1016/j.cie.2018.06.014

[3] Ben-Tal, A., El Ghaoui, L., and Nemirovski, A. (2009). Robust optimization. Princeton University Press.

[4] Bertsimas, D., and Sim, M. (2004). The price of robustness. Operations Research, 52(1), 35-53. doi: $10.1287 /$ opre.1030.0065

[5] Bertsimas, D., Brown, D. B., and Caramanis, C. (2011). Theory and applications of robust optimization. SIAM Review, 53(3), 464-501. doi: 10.1137/080734510

[6] Chica, M., Bautista, J., and de Armas, J. (2019). Benefits of robust multiobjective optimization for flexible automotive assembly line balancing. Flexible Services and Manufacturing Journal, 31(1), 75-103. doi: 10.1007/s10696-018-9309-y

[7] Erel, E., Sabuncuoglu, I., and Sekerci, H. (2005). Stochastic assembly line balancing using beam search. International Journal of Production Research, 43(7), 1411-1426. doi: 10.1080/00207540412331320526

[8] Foroughi, A., and Gökçen, H. (2019). A multiple rule-based genetic algorithm for costoriented stochastic assembly line balancing problem. Assembly Automation, 39(1), 124-139. doi: 10.1108/AA-03-2018-050

[9] Gurevsky, E., Hazır, Ö., Battaïa, O., and Dolgui, A. (2013). Robust balancing of straight assembly lines with interval task times. Journal of the Operational Research Society, 64(11), 1607-1613. doi: $10.1057 /$ jors. 2012.139

[10] Hazır, Ö., and Dolgui, A. (2013). Assembly line balancing under uncertainty: Robust optimization models and exact solution method. Computers $\&$ Industrial Engineering, 65(2), 261-267. doi: 10.1016/j.cie.2013.03.004

[11] Hazır, Ö., and Dolgui, A. (2015). A decomposition based solution algorithm for U-type assembly line balancing with interval data. Computers $\&$ Operations Research, 59, 126-131. doi: 10.1016/j.cor.2015.01.010

[12] Li, B. H., Xiao, T., Zhang, L., and Xu, W. (2009). Robust balancing of mixed model assembly line. COMPEL-The international journal for computation and mathematics in electrical and electronic engineering, 28(6), 1489-1502. doi: 10.1108/03321640910992038

[13] Moreira, M. C. O., Cordeau, J. F., Costa, A. M., and Laporte, G. (2015). Robust assembly line balancing with heterogeneous workers. Computers $\&$ Industrial Engineering, 88, 254-263. doi: 10.1016/j.cie.2015.07.004

[14] Nakade, K., Ito, A., and Ali, S. M. (2015). U-shaped Assembly Line Balancing with temporary workers. International Journal of Industrial Engineering: Theory, Applications and Practice, 21, 134-146. https://journals.sfu.ca/ijietap/index.php/ijie/article/view/1211/0

[15] Oksuz, M. K., Buyukozkan, K., and Satoglu, S. I. (2017). U-shaped assembly line worker assignment and balancing problem: A mathematical model and two meta-heuristics. Computers $\&$ Industrial Engineering, 112, 246-263. doi: 10.1016/j.cie.2017.08.030 
[16] Özcan, U. (2010). Balancing stochastic two-sided assembly lines: A chance-constrained, piecewiselinear, mixed integer program and a simulated annealing algorithm. European Journal of Operational Research, 205(1), 81-97. doi: 10.1016/j.ejor.2009.11.033

[17] Özçelik, G., Yılmaz, Ö. F., and Yeni, F. B. (2020). Robust optimisation for ripple effect on reverse supply chain: An industrial case study. International Journal of Production Research, 1-20. doi: 10.1080/00207543.2020.1740348

[18] Pereira, J. (2018). The robust (minmax regret) assembly line worker assignment and balancing problem. Computers \& Operations Research, 93, 27-40. doi: 10.1016/j.cor.2018.01.009

[19] Pereira, J., and Álvarez-Miranda, E. (2018). An exact approach for the robust assembly line balancing problem. Omega, 78, 85-98. doi: 10.1016/j.omega.2017.08.020

[20] Samouei, P., and Ashayeri, J. (2019). Developing optimization \& robust models for a mixedmodel assembly line balancing problem with semi-automated operations. Applied Mathematical Modelling, 72, 259-275. doi: 10.1016/j.apm.2019.02.019

[21] Soyster, A. L. (1973). Convex programming with set-inclusive constraints and applications to inexact linear programming. Operations Research, 21(5), 1154-1157. doi: 10.1287/opre.21.5.1154

[22] Toloo, M., and Mensah, E. K. (2019). Robust optimization with nonnegative decision variables: a DEA approach. Computers \& Industrial Engineering, 127, 313-325. doi: 10.1016/j.cie.2018.10.006

[23] Urban, T. L. (1998). Note. Optimal balancing of U-shaped assembly lines. Management Science, 44(5), 738-741. doi: 10.1287/mnsc.44.5.738

[24] Yilmaz, Ö. F., Özçelik, G., and Yeni, F. B. (2020). Ensuring sustainability in the reverse supply chain in case of the ripple effect: A two-stage stochastic optimization model. Journal of Cleaner Production, 124548. doi: 10.1016/j.jclepro.2020.124548 